\title{
Scaling and design of high-energy laser plasma electron acceleration
}

\author{
Kazuhisa Nakajima, Hyung Taek Kim, Tae Moon Jeong, and Chang Hee Nam \\ Center for Relativistic Laser Science, Institute for Basic Science (IBS), Gwangju 500-712, Korea \\ (Received 19 February 2014; revised 25 August 2014; accepted 4 February 2015)
}

\begin{abstract}
Recently there has been great progress in laser-driven plasma-based accelerators by exploiting high-power lasers, where electron beams can be accelerated to multi-GeV energy in a centimeter-scale plasma due to the laser wakefield acceleration mechanism. While, to date, worldwide research on laser plasma accelerators has been focused on the creation of compact particle and radiation sources for basic sciences, medical and industrial applications, there is great interest in applications for high-energy physics and astrophysics, exploring unprecedented high-energy frontier phenomena. In this context, we present an overview of experimental achievements in laser plasma acceleration from the perspective of the production of $\mathrm{GeV}$-level electron beams, and deduce the scaling formulas capable of predicting experimental results self-consistently, taking into account the propagation of a relativistic laser pulse through plasma and the accelerating field reduction due to beam loading. Finally, we present design examples for 10-GeV-level laser plasma acceleration, which is expected in near-term experiments by means of petawatt-class lasers.
\end{abstract}

Keywords: electron beam loading; GeV-level electron beam acceleration; laser plasma (wakefield) accelerators; petawatt-class lasers; propagation of relativistic laser pulses in plasma

\section{Introduction}

In this decade, active research has been carried out on the laser plasma acceleration concept ${ }^{[1]}$ in order to achieve highenergy, high-quality electron beams with GeV energy in a cm-scale plasma ${ }^{[2-6]}, 1 \%$-level energy spread ${ }^{[7]}, 1-\mathrm{mm}^{-}$ mrad-level transverse emittance ${ }^{[8]}$ and 1-fs-level bunch duration ${ }^{[9]}$, ensuring that the stability of reproduction is as high as that of present high-power ultrashort-pulse lasers ${ }^{[10]}$. Recently, staged laser plasma acceleration ${ }^{[11,12]}$ has been successfully demonstrated in conjunction with ionizationinduced injection ${ }^{[13-15]}$. Based on recent results on laser plasma acceleration experiments and large-scale particlein-cell (PIC) simulations ${ }^{[16-18]}$, design consideration and feasibility studies on applications for high-energy frontier colliders with TeV-range center-of-mass energy have been carried out ${ }^{[19,20]}$. In this context, state-of-the-art PW-class lasers allow us to study the feasibility of laser plasma accelerators toward the $10-100-\mathrm{GeV}$ range in a full-scale experiment. For example, a large-scale experiment ${ }^{[21]}$ is proposed to implement the demonstration of $100-\mathrm{GeV}$ electron beam acceleration by means of a laser plasma accelerator

Correspondence to: K. Nakajima, Center for Relativistic Laser Science, Institute for Basic Science (IBS), Gwangju 500-712, Korea.

Email: naka115@dia-net.ne.jp driven with a multi-PW laser capable of delivering 3.5-kJ, 500-fs pulses ${ }^{[22]}$. This capability allows us to explore laser plasma acceleration operated in the entire laser wakefield regime, from the linear regime to the nonlinear bubble regime. Such a large-scale laser plasma accelerator may comprise a gas jet or a short gas cell, which acts as an injector, followed by a long, uniform, low-density plasma or preformed plasma channel (plasma waveguide) that acts as an accelerating medium. In order to implement this project, we need to strongly corroborate design formulas that scale experimental results properly.

We organize the remainder of this paper as follows. Section 2 presents an overview of progress and recent achievements in laser plasma electron acceleration. Section 3 considers the energy scaling of laser wakefield acceleration for the self-guided and channel-guided cases in both the quasi-linear and the nonlinear bubble regimes as well as the comparison of such scaling formulas with recent experimental results on multi-GeV electron acceleration. Section 4 presents the design formulas and examples of $10-\mathrm{GeV}$ level laser wakefield accelerators as well as a comparison with the result from a three-dimensional full-scale PIC simulation $^{[16]}$. 


\section{Overview of laser plasma electron acceleration}

Laser-driven plasma-based accelerators have evolved from a groundbreaking concept by Tajima and Dawson ${ }^{[1]}$ into the reality of next-generation particle accelerator technologies. Relativistic electron beams from ultraintense laser plasma interactions can be conceived to be compact particle accelerators, inspiring a wide range of applications of unique particle beam and radiation sources such as $\mathrm{THz}^{[23]}$ and betatron $\mathrm{x}$-ray radiation ${ }^{[24]}$. Furthermore, it is envisaged that laser plasma accelerators will downsize large-scale particle accelerators such as $\mathrm{x}$-ray free-electron lasers ${ }^{[25-28]}$ and high-energy frontier colliders ${ }^{[19,20]}$ to a realistic extent in both dimensions and costs.

In fact, there has been significant experimental progress in laser wakefield acceleration of electron beams since the incipient experiments on laser wakefield accelerators successfully demonstrated ultrahigh gradient acceleration of the order of $100 \mathrm{GeV} \mathrm{m}^{-1}$, using chirped pulse amplification lasers with 10-TW class peak power and 1 ps pulse duration ${ }^{[29,30]}$. Such experiments are characterized in terms of the self-modulated wakefield regime ${ }^{[31]}$, where the laser power should be higher than the critical power for relativistic self-focusing and the laser pulse duration is longer than the plasma period. In this regime, the laser pulse undergoes temporal intensity modulation and self-guiding through nonlinear interactions with the plasma, so that largeamplitude plasma waves are resonantly excited. Ultimately, wave breakings occur, generating relativistic electrons to be randomly trapped and accelerated by wakefields throughout the acceleration distance. Therefore, electron beams produced from single-stage experiments showed energy spectra with $100 \%$ energy spread, as characterized by a Maxwellian distribution with the highest energy tail reaching at most $100 \mathrm{MeV}^{[32]}$. The energy gain of accelerated electrons should be determined by the acceleration distance, which is restricted due to dephasing of electrons with respect to the correct acceleration phase of the wakefield and due to depletion of the laser pulse energy. For most experiments using a supersonic gas jet, the acceleration distance extends only to a few $\mathrm{mm}$, so that the energy gain is limited to the order of $200 \mathrm{MeV}^{[33]}$.

For many practical applications of electron beams, quality, stability and controllability of the beam performance such as energy, energy spread, emittance and charge are indispensable in addition to compact and robust features of accelerators. In this context, breakthrough experiments ${ }^{[34-36]}$ have succeeded in producing high-quality electron beams, so-called quasi-monoenergetic beams, with ultrashort pulses, small energy spread and low emittance. Quasi-monoenergetic electron beams have been obtained from the use of ultrashort laser pulses with durations of the order of several tens of femtoseconds and by controlling the plasma density precisely to make the dephasing length long enough to exceed the acceleration distance. Under these conditions, once the plasma electrons expelled by the ponderomotive force (radiation pressure) of the laser pulse form a plasma cavity called a 'bubble', then some of them are self-injected into the wakefield by a wavebreaking or restoring force exerted by an ion channel remaining unshielded behind the laser pulse. As a result of beam loading of trapped electrons, the nonlinear wakefield amplitude inside the bubble is reduced below the trapping threshold. Consequently, electrons trapped in the wakefield undergo the processes of acceleration and bunching toward the wave crest to increase their energy and brightness unless the acceleration distance exceeds the dephasing length. This is a scenario of quasi-monoenergetic electron beam acceleration, based on the self-injection mechanism in the bubble regime, which is visually shown by multidimensional PIC simulations ${ }^{[37,38]}$.

Although self-injection is a robust method relying on selffocusing, self-compression of the laser pulse and expansion of the bubble ${ }^{[39]}$ which occur during the propagation of relativistic laser pulses, initially heated (accelerated) electrons with large transverse momentum are injected into nonlinear wakefields that excite betatron oscillation of accelerated electrons due to the strong focusing field. Hence, if the self-injection and the deterioration of beam quality are suppressed, high-quality electron beams can be produced by controlled injection schemes such as colliding optical injection ${ }^{[40,41]}$, density-transition injection ${ }^{[42]}$ or density down-ramp ${ }^{[43]}$ and ionization-induced injection ${ }^{[13-15]}$ in the quasi-linear regime of wakefields driven by a laser pulse with a moderate intensity. These injection schemes provide us with high-quality electron beam injectors for the front end of multi-stage high-energy accelerators. As a simplest case, two-stage laser plasma acceleration has been successfully demonstrated in combination with ionization-induced injection $^{[11,12]}$.

For laser plasma acceleration reaching GeV-level energies, it is essential to propagate intense laser pulses over a centimeter-scale distance in underdense plasma. For this purpose, a preformed plasma density channel with a parabolic radial distribution ${ }^{[44]}$ has been developed for guiding a laser beam over many Rayleigh lengths without diffraction which limits the acceleration distance to a few $\mathrm{mm}$ in a uniform plasma. Plasma density channels stabilize the propagation of relativistically intense laser pulses under a matched condition, preventing laser plasma nonlinear instabilities, such as filamentation and hosing which often occur in self-guiding ${ }^{[4,46]}$. Therefore, the concept of channel-guided laser wakefield accelerators has been a longstanding proposal ${ }^{[47]}$. Employing a centimeter-scale plasma waveguide, experiments on $\mathrm{GeV}$-level electron acceleration have been carried out with a gas-filled or ablative discharge capillary ${ }^{[2,7,8]}$ to demonstrate quasi-monoenergetic electron beams. Experimental progress beyond $1 \mathrm{GeV}$ has 
been reported in experiments making use of a 100-TW class laser and a centimeter-scale capillary discharge plasma waveguide $^{[4]}$ or a gas cell relying on self-guiding ${ }^{[3]}$. During the last two decades, a number of laser plasma accelerator experiments have been carried out under various conditions. Comparing these data with theoretical laser wakefield acceleration models, it may be useful to find a correct scaling law capable of predicting energy gain, accelerated electron charge and the required laser and plasma conditions.

\section{Energy scaling of laser wakefield acceleration in the relativistic regime}

\subsection{Propagation of relativistic laser pulses in plasma}

The wave equation for the normalized vector potential describing the evolution of a laser pulse with laser wavelength $\lambda_{L}$ and duration $\tau_{L}$ (full width at half maximum, FWHM) in a plasma channel can be written as ${ }^{[48]}$

$$
\left(\nabla^{2}-\frac{\partial^{2}}{c^{2} \partial t^{2}}\right) \mathbf{a}=k^{2}\left(1-\eta^{2}\right) \mathbf{a},
$$

where $\mathbf{a} \equiv e \mathbf{A} / m_{e} c^{2}$ is the vector potential $\mathbf{A}$ of the laser pulse normalized with respect to the electron rest energy $m_{e} c^{2}$, satisfying the Coulomb gauge $\nabla \cdot \mathbf{a}=0$, and $k=$ $\omega / c=2 \pi / \lambda_{L}$ is the free-space wavenumber along the propagation direction. The (squared) refractive index for linearly polarized electromagnetic waves in the long-pulse limit $\left(c k_{p} \tau_{L} \gg 1\right)$ is given by ${ }^{[49]}$

$$
\eta^{2}(r, z)=1-\frac{k_{p}^{2}}{k^{2} \gamma_{L}}\left[1+\frac{1}{k_{p}^{2}} \nabla_{\perp}^{2} \gamma_{L}+\frac{\Delta n}{n_{0}} \frac{r^{2}}{r_{0}^{2}}\right],
$$

where $k_{p}=\omega_{p} / c=\left(4 \pi r_{e} n_{0}\right)^{1 / 2}$ is the plasma wavenumber evaluated with the unperturbed on-axis density $n_{0}$ and the classical electron radius $r_{e}=e^{2} / m c^{2}$, and $\gamma_{L}=(1+$ $\left.a^{2} / 2\right)^{1 / 2}$ is the relativistic factor of the laser intensity for linear polarization. In Equation (2), the first term represents free-space propagation, and the three terms in the square brackets correspond to relativistic self-focusing, ponderomotive channeling and a preformed plasma channel with a parabolic density distribution of the form $n(r)=n_{0}[1+$ $\left.\left(\Delta n / n_{0}\right)\left(r^{2} / r_{L}^{2}\right)\right]$, where $r_{L}$ is the laser spot radius and $\Delta n$ is the channel depth. Analysis of the wave equation with the standard paraxial form provides the matched spot radius $r_{m}$ under the condition of a beam propagating with a constant spot size $r_{L}$, i.e., $k_{p} r_{m}=k_{p} r_{L}=R_{m}$, given by ${ }^{[21]}$

$$
\begin{aligned}
k_{p}^{2} r_{m}^{2} \equiv & R_{m}^{2}\left(a_{0}^{2}, \Delta n / n_{0}\right) \\
= & 2 \ln \left(\gamma_{L 0}\right)\left\{\gamma_{L 0}-1-2 \ln \left(\left(1+\gamma_{L 0}\right) / 2\right)\right. \\
& +\left(\Delta n / n_{0}\right)\left[1-\gamma_{L 0}+\ln \left(\left(1+\gamma_{L 0}\right) / 2\right)\right. \\
& \left.\left.+\left(a_{0}^{2} / 4\right)_{4} F_{3}\left(1 / 2,1,1,1 ; 2,2,2 ;-a_{0}^{2} / 2\right)\right]\right\}^{-1},
\end{aligned}
$$

where $\gamma_{L 0}=\left(1+a_{0}^{2} / 2\right)^{1 / 2}$ is the relativistic factor for a Gaussian laser beam with peak amplitude $a_{0}$ and ${ }_{p} F_{q}$ denotes the generalized hypergeometric series of order $q$ and class $q-p+1$.

Under the matched condition that no phase shift of the laser pulse occurs, the group velocity is written as $\beta_{g}^{2} \approx$ $1-k_{p}^{2} /\left(\kappa_{\mathrm{ch}} k^{2}\right)$, where a correction factor for the group velocity is defined as ${ }^{[21]}$

$$
\begin{gathered}
\kappa_{\mathrm{ch}}\left(a_{0}^{2}, \Delta n / n_{0}\right)=\frac{a_{0}^{2}}{8}\left[\gamma_{L 0}-1-\ln \left(\left(1+\gamma_{L 0}\right) / 2\right)\right. \\
\left.+\frac{a_{0}^{2}}{8} \frac{\Delta n}{n_{0}}{ }_{4} F_{3}\left(1 / 2,1,1,1 ; 2,2,2 ;-a_{0}^{2} / 2\right)\right]^{-1} .
\end{gathered}
$$

Under the matched condition for self-guiding, the spot radius and the group velocity correction factor are given by Equations (3) and (4), respectively, where the channel depth is set to be $\Delta n=0$.

\subsection{Laser plasma acceleration in the quasi-linear regime}

In the linear laser wakefield with the accelerating field $E_{z}=$ $E_{z 0} \cos \Psi$, the equations of the longitudinal motion of an electron with the normalized velocity $\beta_{z}=v_{z} / c \approx 1$ and electron energy $\gamma=E_{e} / m_{e} c^{2}$ are given by ${ }^{[50]}$

$$
\begin{gathered}
\frac{d \gamma}{d z}=k_{p} \frac{E_{z 0}}{E_{0}} \cos \Psi \quad \text { and } \\
\frac{d \Psi}{d z}=k_{p}\left(1-\frac{\beta_{p}}{\beta_{z}}\right) \approx \frac{k_{p}}{2 \gamma_{g}^{2}},
\end{gathered}
$$

where $\Psi=k_{p}\left(z-v_{p} t\right)+\Psi_{0}$ is the phase of the plasma wave, $E_{0}=m c \omega_{p} / e$ is the non-relativistic wave-breaking field approximately given by $E_{0} \approx 96\left[\mathrm{GV} \mathrm{m}^{-1}\right]$ $\left(n_{e} / 10^{18}\left[\mathrm{~cm}^{-3}\right]\right)^{1 / 2}, \beta_{p}=v_{p} / c \approx v_{g} / c=\beta_{g}$ is the phase velocity $v_{p}$ of the plasma wave normalized to $c$, and $\gamma_{g}=\left(1-\beta_{g}^{2}\right)^{-1 / 2} \gg 1$ is assumed. By integrating Equations (5), the energy and phase of the electron can be calculated as

$$
\begin{gathered}
\gamma(z)=\gamma_{0}+2 \gamma_{g}^{2} \frac{E_{z 0}}{E_{0}}\left[\sin \Psi(z)-\sin \Psi_{0}\right] \text { and } \\
\Psi(z) \approx \frac{k_{p} z}{2 \gamma_{g}^{2}}+\Psi_{0} .
\end{gathered}
$$

Setting the initial electron phase $\Psi_{0}=0$ at $z=0$, the maximum energy gain is given by

$$
\Delta \gamma_{\max }=\gamma_{\max }-\gamma_{0}=2 \gamma_{g}^{2} \frac{E_{z 0}}{E_{0}}
$$

at $k_{p} z=\pi \gamma_{g}^{2}$ or $z=\lambda_{p} \gamma_{g}^{2} / 2$. As shown from Equations (6), setting $\Psi_{0}=-\pi / 2$, the maximum energy gain reaches $\Delta \gamma_{\max }=4 \gamma_{g}^{2} E_{z 0} / E_{0}$ at $k_{p} z=2 \pi \gamma_{g}^{2}$ or $z=\lambda_{p} \gamma_{g}^{2}$. However, 
electrons undergo both acceleration and focusing only for the phase $0 \leqslant \Psi \leqslant \pi / 2$. Hence, we define the dephasing length as $L_{\mathrm{dp}}=\lambda_{p} \gamma_{g}^{2} / 2$. Considering a driving laser pulse of normalized intensity $a_{0}^{2}$ moving in a plasma channel with channel depth $\Delta n$ at the group velocity $\beta_{g}=v_{g} / c$ with the corresponding relativistic factor $\gamma_{g}^{2}=\left(1-\beta_{g}^{2}\right)^{-1} \approx$ $\kappa_{\mathrm{ch}}\left(\omega^{2} / \omega_{p}^{2}\right)=\kappa_{\mathrm{ch}}\left(n_{c} / n_{e}\right)=\kappa_{\mathrm{ch}} \gamma_{g 0}^{2}$, where $\gamma_{g 0}=\omega / \omega_{p}$ is the relativistic factor for the group velocity in a uniform plasma and its correction factor $\kappa_{\mathrm{ch}}$ is given by Equation (4), the maximum energy gain and the dephasing length are written as $\Delta \gamma_{\max }=2 \kappa_{\mathrm{ch}} \gamma_{g 0}^{2}\left(E_{z 0} / E_{0}\right)$ and $L_{\mathrm{dp}}=\left(\lambda_{p} / 2\right) \kappa_{\mathrm{ch}} \gamma_{g o}^{2}$, respectively. In the limit of $a_{0}^{2} \ll 1, \kappa_{\mathrm{ch}} \approx\left(1+\Delta n / n_{0}\right)^{-1}$. In the quasi-linear regime, taking the beam loading effect into account, the maximum accelerating field driven by a Gaussian laser pulse is given by ${ }^{[20]}$

$$
\frac{E_{z 0}}{E_{0}} \simeq \sqrt{\pi} \alpha a_{0}^{2}\left(\frac{k_{p} \sigma_{L}}{4}\right) \exp \left(-\frac{k_{p}^{2} \sigma_{L}^{2}}{4}\right) \approx 0.38 \alpha a_{0}^{2},
$$

where $\alpha$ denotes a factor of accelerating field reduction due to the beam loading effect and $\sigma_{L}$ is the rms pulse length of the Gaussian temporal profile with the FWHM length $c \tau_{L} \sim$ $0.375 \lambda_{p}$ for $k_{p} \sigma_{L}=\sqrt{2}$.

\subsection{Laser plasma acceleration in the bubble regime}

Previous laser plasma acceleration experiments that successfully demonstrated the production of quasi-monoenergetic electron beams with narrow energy spread have been elucidated in terms of self-injection and an acceleration mechanism in the bubble regime ${ }^{[37,38]}$. In these experiments, electrons are self-injected into a nonlinear wake, often referred to as a bubble, i.e., a cavity void of plasma electrons consisting of a spherical ion column surrounded by a narrow electron sheath, formed behind the laser pulse instead of a periodic plasma wave in the linear regime. The phenomenological theory of a nonlinear wakefield in the bubble regime describes the accelerating wakefield $E_{z}(\xi) / E_{0} \approx(1 / 2) k_{p} \xi$ in the bubble frame moving in the plasma with velocity $v_{B}$, i.e., $\xi=z-v_{B} t$. In the bubble (blowout) regime for $a_{0} \geqslant 2$, since the electron-evacuated cavity shape is determined by balancing the Lorentz force of the ion sphere exerted on the electron sheath with the ponderomotive force of the laser pulse, the bubble radius $R_{B}$ is approximately given as $k_{p} R_{B} \approx 2 \sqrt{a_{0}}$ (Ref. [38]). Thus, the maximum accelerating field is obtained as $E_{z 0} / E_{0}=(1 / 2) \alpha k_{p} R_{B}$, where $\alpha$ represents a factor that takes into account the difference between the simulation and theoretical estimation, and more significantly the accelerating field reduction due to the beam loading effects.
In self-guided laser wakefield acceleration, where a driving laser pulse propagates by means of self-channeling, the equations of longitudinal motion of an electron are approximately written as ${ }^{[21]}$

$$
\begin{aligned}
& \frac{d \gamma}{d z}=k_{p} \frac{E_{z 0}}{E_{0}}\left(1-\frac{\xi}{R_{B}}\right)=\frac{1}{2} \alpha k_{p}^{2} R_{B}\left(1-\frac{\xi}{R_{B}}\right) \\
& \text { and } \frac{d \xi}{d z}=1-\frac{\beta_{B}}{\beta_{z}} \approx 1-\beta_{B} \approx \frac{3}{2 \gamma_{g}^{2}},
\end{aligned}
$$

where $\xi=z-v_{B} t\left(0 \leqslant \xi \leqslant R_{B}\right)$ is the longitudinal coordinate of the bubble frame moving at the velocity $v_{B}=$ $c \beta_{B} \approx v_{g}-v_{\text {etch }}$ and taking into account the diffraction at the laser front that etches back at the velocity $v_{\text {etch }} \simeq$ $c\left(\omega_{p} / \omega\right)^{2}$ (Ref. [38]). Integrating Equations (5), the energy and phase of the electron can be calculated as

$$
\begin{aligned}
& \gamma(z)=\gamma_{0}+\frac{1}{3} \alpha \gamma_{g}^{2} k_{p}^{2} R_{B} \xi(z)\left(1-\frac{1}{2} \frac{\xi(z)}{R_{B}}\right) \\
& \text { and } \xi(z)=\frac{3}{2} \frac{z}{\gamma_{g}^{2}},
\end{aligned}
$$

where $\gamma_{0}=\gamma(0)$ is the injection energy. Hence, the maximum energy gain is obtained at $\xi=R_{B}$ as

$$
\begin{aligned}
\Delta \gamma_{\max } & =\gamma_{\max }-\gamma_{0} \approx \frac{1}{6} \alpha \gamma_{g}^{2} k_{p}^{2} R_{B}^{2} \approx \frac{2}{3} \alpha a_{0} \gamma_{g}^{2} \\
& =\frac{2}{3} \alpha \kappa_{\text {self }} a_{0} \frac{n_{c}}{n_{e}} .
\end{aligned}
$$

The dephasing length $L_{\mathrm{dp}}$ for the self-guided bubble regime is given by

$$
k_{p} L_{\mathrm{dp}} \approx \frac{2}{3} k_{p} R_{B} \gamma_{g}^{2}=\frac{4}{3} \sqrt{a_{0}} \kappa_{\mathrm{self}} \frac{n_{c}}{n_{e}},
$$

i.e.,

$$
\begin{aligned}
L_{\mathrm{dp}} & \simeq \frac{4}{3 \pi} \lambda_{L} \sqrt{a_{0}} \kappa_{\mathrm{self}}\left(\frac{n_{c}}{n_{e}}\right)^{3 / 2} \\
& \approx 24.7[\mathrm{~mm}] \sqrt{a_{0}} \kappa_{\mathrm{self}}\left(\frac{0.8 \mu \mathrm{m}}{\lambda_{L}}\right)^{2}\left(\frac{10^{18} \mathrm{~cm}^{-3}}{n_{e}}\right)^{3 / 2}
\end{aligned}
$$

while the pump depletion length due to pulse-front erosion is given by

$$
L_{\mathrm{pd}} \approx c \tau_{L} \frac{n_{c}}{n_{e}},
$$

where $\kappa_{\text {self }} \equiv \kappa_{\mathrm{ch}}\left(a_{0}^{2}, 0\right)$ is the correction factor of the group velocity for the self-guided pulse in Equation (4).

For a driving laser pulse propagating in a plasma channel, the equations of electron motion are given by setting $v_{B}=$ $c \beta_{B} \approx v_{g}$ in Equations (5), i.e., $d \xi / d z \approx 1-\beta_{B} \approx 1 / 2 \gamma_{g}^{2}$. 
Table 1. Parameters of experiments on $\mathrm{GeV}$-class laser wakefield acceleration.

\begin{tabular}{|c|c|c|c|c|c|c|c|c|c|c|}
\hline Ref. & $\begin{array}{l}n_{e} / 10^{18} \\
\left(\mathrm{~cm}^{-3}\right)\end{array}$ & $\begin{array}{l}\Delta n / n_{e} \\
(\%)\end{array}$ & $\begin{array}{l}L_{\mathrm{acc}} \\
(\mathrm{mm})\end{array}$ & $\begin{array}{l}P_{L} \\
\text { (TW) }\end{array}$ & $P / P_{c}$ & $\begin{array}{l}r_{L} \\
(\mu \mathrm{m})\end{array}$ & $\begin{array}{l}\tau_{L} \\
\text { (fs) }\end{array}$ & $a_{0}$ & $\begin{array}{l}E_{\mathrm{ex}} \\
(\mathrm{GeV})\end{array}$ & $\begin{array}{l}\Delta E / E \\
(\%)\end{array}$ \\
\hline$\overline{[51]}$ & 5.7 & 0 & 8.7 & 182 & 35 & 19 & 55 & 3.9 & 0.8 & 12 \\
\hline [52] & 3 & 0 & 8 & 65 & 6.6 & 15 & 60 & 2.8 & 0.72 & 14 \\
\hline [3] & 1.3 & 0 & 13 & 110 & 4.9 & 15 & 60 & 3.8 & 1.45 & $\sim 100$ \\
\hline [11] & $5.7+2.5$ & 0 & $1+3$ & 45 & 3.8 & 16 & 40 & 2.3 & 0.8 & 25 \\
\hline [12] & 3 & 0 & $3+5$ & 40 & 4.1 & 15 & 60 & 2.3 & 0.46 & 5 \\
\hline [5] & 0.48 & 0 & 67 & 625 & 18 & 50 & 160 & 3 & 2 & 10 \\
\hline [6] & 2.1 & 0 & 4 & 212 & 15 & 21 & 60 & 3.7 & 0.35 & $\sim 25$ \\
\hline [6] & 1.3 & 0 & 10 & 212 & 8 & 21 & 60 & 3.7 & 0.87 & $\sim 30$ \\
\hline [6] & $2+0.8$ & 0 & $4+10$ & 212 & 5.8 & 21 & 60 & 3.7 & 3 & $\sim 30$ \\
\hline [2] & 4.3 & $<5$ & 33 & 40 & 5.8 & 25 & 37 & 1.4 & 1 & 5.9 \\
\hline [2] & 3.5 & $<5$ & 33 & 12 & 1.4 & 25 & 80 & 0.75 & 0.5 & 13 \\
\hline [8] & 8.4 & $<5$ & 15 & 18 & 5.1 & 23 & 42 & 0.84 & 0.5 & 2.5 \\
\hline [7] & 1.9 & $<5$ & 40 & 24 & 1.55 & 17 & 27 & 1.7 & 0.56 & 2.8 \\
\hline [53] & 1.8 & $<5$ & 30 & 32 & 1.96 & 22 & 80 & 1.4 & 0.52 & 5 \\
\hline [4] & 3.1 & $<5$ & 40 & 130 & 13.7 & 21 & 55 & 3 & 1.8 & $\sim 50$ \\
\hline
\end{tabular}

Hence, the maximum energy gain is

$$
\begin{aligned}
\Delta \gamma_{\max } & =\gamma_{\max }-\gamma_{0} \approx \frac{1}{2} \alpha \gamma_{g}^{2} k_{p}^{2} R_{B}^{2} \approx 2 \alpha a_{0} \gamma_{g}^{2} \\
& =2 \alpha \kappa_{\mathrm{ch}} a_{0} \frac{n_{c}}{n_{e}}
\end{aligned}
$$

and the dephasing length $L_{\mathrm{dp}}$ is

$$
k_{p} L_{\mathrm{dp}} \approx 2 k_{p} R_{B} \gamma_{g}^{2}=4 \sqrt{a_{0}} \kappa_{\mathrm{ch}} \frac{n_{c}}{n_{e}},
$$

where $\kappa_{\mathrm{ch}}$ is given by Equation (4). For channel-guided laser plasma acceleration, the pump depletion length, at which the total field energy becomes half of the initial laser energy, is given by

$$
L_{\mathrm{pd}} \approx \frac{\sqrt{\pi}}{2 \alpha^{2}} a_{0} \sigma_{L} \frac{n_{c}}{n_{e}}=\frac{1}{4} \sqrt{\frac{\pi}{\ln 2}} \frac{a_{0} c \tau_{L}}{\alpha^{2}} \frac{n_{c}}{n_{e}},
$$

where $\sigma_{L}=c \tau_{L} /(2 \sqrt{\ln 2}) \approx 0.6 c \tau_{L}$ is the rms pulse length.

The matched power $P_{m}$ corresponding to the matched spot size $r_{L}$ is calculated as

$$
P_{L}=\frac{k_{p}^{2} r_{L}^{2} a_{0}^{2}}{32} P_{c}
$$

where $P_{c}=17 n_{c} / n_{0}[\mathrm{GW}]$ is the critical power for the relativistic self-focusing at the plasma density $n_{0}$ and the required pulse energy is $U_{L}=P_{L} \tau_{L}$.

\subsection{Beam loading effects}

In laser wakefield acceleration, an accelerated electron beam induces its own wakefield and cancels the laser-driven wakefield. Assuming the beam loading efficiency $\eta_{b} \equiv 1-$ $E_{z}^{2} / E_{M}^{2}$ defined by the fraction of the plasma wave energy absorbed by particles of the bunch with the rms radius $\sigma_{b}$, the beam-loaded field is given by $E_{z}=\sqrt{1-\eta_{b}} E_{M}=\alpha E_{M}$, where $E_{M}$ is the accelerating field without beam loading, given by $E_{M} \approx a_{0}^{1 / 2} E_{0}$ for the bubble regime $a_{0} \geqslant 2$. Thus, the loaded charge is calculated as ${ }^{[20]}$

$$
\begin{aligned}
Q_{b} & \simeq \frac{e}{4 k_{L} r_{e}} \frac{\eta_{b} k_{p}^{2} \sigma_{b}^{2}}{1-\eta_{b}} \frac{E_{z}}{E_{0}}\left(\frac{n_{c}}{n_{e}}\right)^{1 / 2} \\
& \approx 76[\mathrm{pC}] \frac{\left(1-\alpha^{2}\right) a_{0}^{1 / 2} k_{p}^{2} \sigma_{b}^{2}}{\alpha}\left(\frac{n_{e}}{10^{18} \mathrm{~cm}^{-3}}\right)^{-1 / 2}
\end{aligned}
$$

Here, the field reduction factor $\alpha$ for accelerating charge $Q_{b}$ in the operating plasma density $n_{e}$ is obtained by solving the equation

$$
\alpha^{2}+B \alpha-1=0
$$

where the coefficient $B$ is defined as

$$
B \equiv \frac{1}{a_{0}^{1 / 2} k_{p}^{2} \sigma_{b}^{2}}\left(\frac{Q_{b}}{76 \mathrm{pC}}\right)\left(\frac{n_{e}}{10^{18} \mathrm{~cm}^{-3}}\right)^{1 / 2}
$$

Thus, the field reduction factor is obtained by

$$
\alpha=\frac{B}{2}\left[\left(1+\frac{4}{B^{2}}\right)^{1 / 2}-1\right] \text {. }
$$

For $B \ll 1$, the field reduction factor becomes $\alpha \approx 1$ and contrarily, for $B \gg 1, \alpha \approx 0$.

\subsection{Comparison with experimental results on GeV-class electron beams}

Table 1 summarizes the parameters for experiments on laser wakefield acceleration driven by a self-guided laser pulse with channel depth $\Delta n / n_{e}=0$ (Refs. [3, 5, 6, 
$11,12,51,52])$ and a channel-guided laser pulse with $\Delta n / n_{e} \neq 0$ (Refs. [2, 4, 7, 8, 53]). Since the maximum energy gain scales as $\Delta \gamma_{\max } \propto n_{c} \propto \lambda_{L}^{-2}$ for a given $a_{0}$, most previous experiments have employed chirped pulse amplification lasers with wavelength $\lambda_{L}=800 \mathrm{~nm}$ and pulse duration $\tau_{L} \leqslant 80 \mathrm{fs}$, except for the case of Ref. [5], where a PW-class laser with wavelength $\lambda_{L}=1057 \mathrm{~nm}$ and $\tau_{L} \sim$ $150 \mathrm{fs}$ was employed. The validity of the energy scaling formulas (Equations (7), (11) and (15)) based on the present analytical methods may be verified by comparison with these experimental results. Figure 1 shows the comparison of measured electron beam energies in laser wakefield acceleration with the energy scaling on the operating plasma density for the self-guided case (Figure 1(a)) in the bubble regime and the channel-guided case (Figure 1(b)) in both the quasilinear regime and the bubble regime. In the energy scaling formulas, we assume that the field reduction due to beam loading and the group velocity correction due to relativistic effects cancel each other out, i.e., $\alpha \kappa_{\text {self }} \sim 1$ or $\alpha \kappa_{\text {ch }} \sim 1$.

For self-guided laser wakefield acceleration, the multi$\mathrm{GeV}$ acceleration results reported in Refs. [5, 6] provide us with informative examples for testing the energy scaling formula (Equation (11)). In Ref. [5], a 625-TW, 160-fs laser pulse with wavelength $\lambda_{L}=1057 \mathrm{~nm}$ is focused onto a $1 / e^{2}$ spot radius of $r_{L}=50 \mu \mathrm{m}$, producing $a_{0}=3.6$ at the entrance of a 7-cm long gas cell with $n_{e}=4.8 \times 10^{17} \mathrm{~cm}^{-3}$. The accelerated electron beam has a quasi-monoenergetic peak at $2.0 \mathrm{GeV}$ with a relative energy spread of $10 \%$ (FWHM), containing a total charge of $540 \mathrm{pC}$ in a bunch. In this case, from Equations (3) and (4), the dimensionless matched spot radius $R_{m}=k_{p} r_{m}$ and the correction factor of the group velocity $\kappa_{\text {self }}=\kappa_{\mathrm{ch}}\left(a_{0}^{2}, 0\right)$ are $R_{m}=2.0$ and $\kappa_{\text {self }}=$ 1.46, respectively, at $a_{0}=3.6$. Thus, since the matched spot radius is $r_{m}=15 \mu \mathrm{m}$, the laser pulse undergoes selffocusing after propagating through the gas cell. Using Equations (20) and (21) and assuming the electron beam size $k_{p} \sigma_{b}=1$, the field reduction factor $\alpha$ is calculated as $\alpha \approx 0.32$ ( $B \approx 2.84$ ). From Equation (13), the dephasing length is $L_{\mathrm{dp}} \approx 118 \mathrm{~mm}$, while from Equation (14), the pump depletion length due to pulse-front erosion is $L_{\mathrm{pd}} \approx$ $100 \mathrm{~mm}$. From Equation (10), the electron beam energy at the accelerator length $z=L_{\text {acc }}$ is estimated as

$$
\begin{aligned}
E_{b}\left(L_{\mathrm{acc}}\right)= & E_{b 0}+m_{e} c^{2} \alpha \sqrt{a_{0}} k_{p} L_{\mathrm{acc}}\left(1-\frac{L_{\mathrm{acc}}}{2 L_{\mathrm{dp}}}\right) \\
\approx & E_{b 0}+96[\mathrm{MeV}] \alpha \sqrt{a_{0}}\left(1-\frac{L_{\mathrm{acc}}}{2 L_{\mathrm{dp}}}\right) \\
& \times\left(\frac{L_{\mathrm{acc}}}{1 \mathrm{~mm}}\right)\left(\frac{n_{e}}{10^{18} \mathrm{~cm}^{-3}}\right)^{1 / 2},
\end{aligned}
$$

where $E_{b 0}$ is the injection beam energy. For $L_{\mathrm{acc}}=70 \mathrm{~mm}$, the beam energy is evaluated to be $E_{b}=2.0 \mathrm{GeV}$. This estimate is in good agreement with the measured beam energy of $2.0 \pm 0.1 \mathrm{GeV}^{[5]}$, taking into account the field reduction factor $\alpha$ due to beam loading.
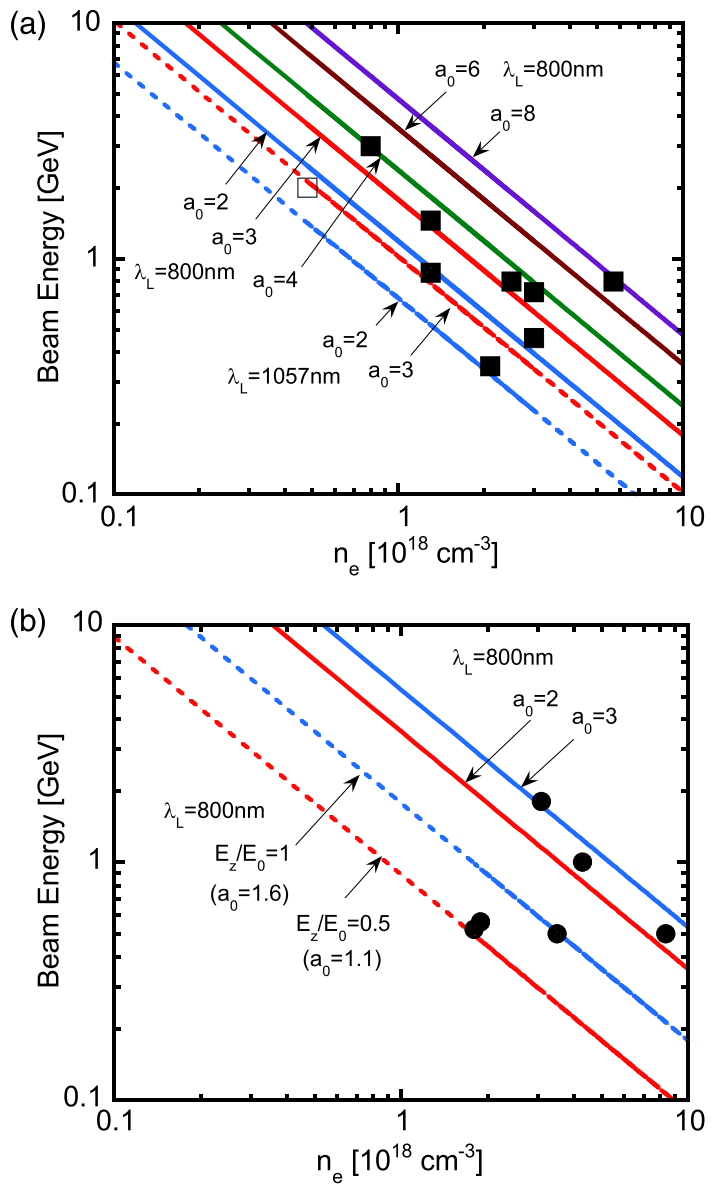

Figure 1. A comparison of measured electron beam energies in laser wakefield acceleration with the energy scaling as a function of the operating plasma density for (a) the self-guided case in the bubble regime at laser wavelengths of $800 \mathrm{~nm}$ (solid line) and $1057 \mathrm{~nm}$ (dashed line) and (b) the channel-guided case in both the quasi-linear regime (dashed line) and the bubble regime (solid line). The experimental data are plotted with filled squares for $\lambda_{L}=800 \mathrm{~nm}$ and the open square for $\lambda_{L}=1057 \mathrm{~nm}$ in (a), and with filled circles for $\lambda_{L}=800 \mathrm{~nm}$ in (b).

In Ref. [6], a 212-TW, 60-fs laser pulse is focused on a $1 / e^{2}$ spot radius of $r_{L}=21 \mu \mathrm{m}$, producing $a_{0}=3.7$ at the entrance of a gas jet for three cases consisting of a 4-mm long single stage with $n_{e}=2.1 \times 10^{18} \mathrm{~cm}^{-3}$, a 10 -mm long single stage with $n_{e}=1.3 \times 10^{18} \mathrm{~cm}^{-3}$, and two stages comprising a 4-mm long injector with $n_{e}=2 \times 10^{18} \mathrm{~cm}^{-3}$ and a $10-\mathrm{mm}$ long accelerator with $n_{e}=0.8 \times 10^{18} \mathrm{~cm}^{-3}$. As shown in Figure 2, the accelerated electron beams for the three cases have peak energies of $0.35,0.87$ and $3 \mathrm{GeV}$, respectively, and total loaded charges of 88,110 and $80 \mathrm{pC}$, respectively. From Equations (3) and (4), the dimensionless matched spot radius $R_{m}=k_{p} r_{m}$ and the correction factor of the group velocity $\kappa_{\text {self }}=\kappa_{\mathrm{ch}}\left(a_{0}^{2}, 0\right)$ are $R_{m}=2.0$ and $\kappa_{\text {self }}=1.48$, respectively, at $a_{0}=3.7$. Since the matched spot radius is $r_{m}=7.3 \mu \mathrm{m}$ for the 4-mm single-stage case, $r_{m}=9.3 \mu \mathrm{m}$ for the $10-\mathrm{mm}$ single-stage case and $r_{m}=7.5 \mu \mathrm{m}$ in the injector jet and $r_{m}=12 \mu \mathrm{m}$ in the accelerator jet for the twostage case, respectively, it is inferred that a laser pulse with 
(a)

(b)

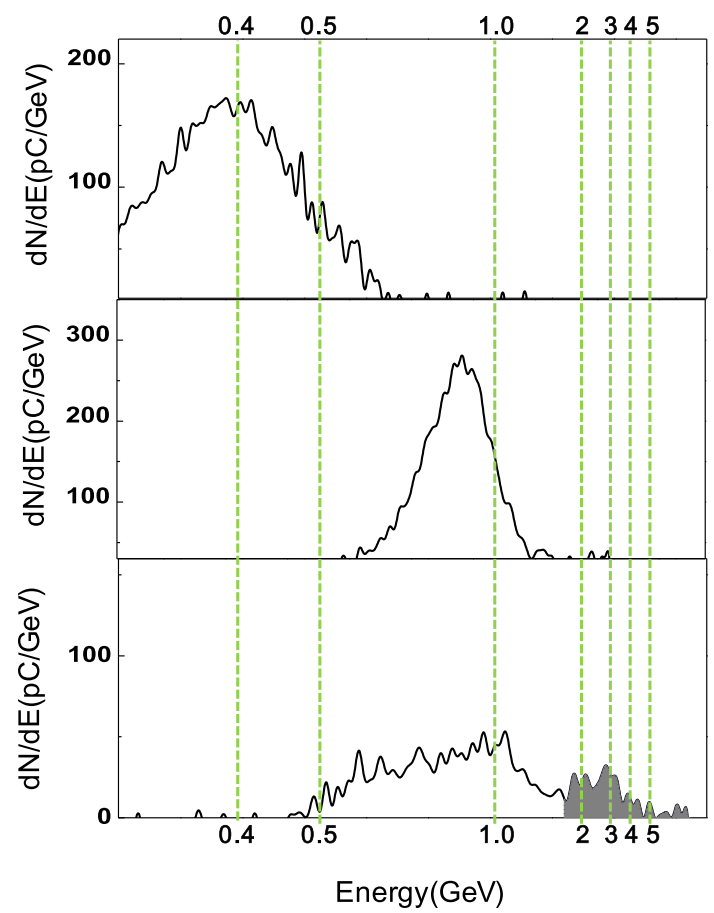

Figure 2. Electron beam energy spectra obtained from the experiment ${ }^{[6]}$, where a $212-\mathrm{TW}, 60-\mathrm{fs}$ laser pulse is focused on a $1 / e^{2}$ spot radius of $r_{L}=21 \mu \mathrm{m}$ producing $a_{0}=3.7$ at the entrance of a gas jet for three cases consisting of (a) a 4-mm long single stage with $n_{e}=2.1 \times 10^{18} \mathrm{~cm}^{-3}$, (b) a $10-\mathrm{mm}$ long single stage with $n_{e}=1.3 \times 10^{18} \mathrm{~cm}^{-3}$ and (c) two stages comprising a 4-mm long injector with $n_{e}=2 \times 10^{18} \mathrm{~cm}^{-3}$ and a 10 -mm long accelerator with $n_{e}=0.8 \times 10^{18} \mathrm{~cm}^{-3}$.

a focused spot radius of $r_{L}=21 \mu \mathrm{m}$ is initially self-focused down to the matched spot radius. Using Equations (20) and (21) and assuming the electron beam size $k_{p} \sigma_{b}=1$, the field reduction factor $\alpha$ is calculated as $\alpha \approx 0.66(B=0.86)$ for the 4-mm single-stage case, $\alpha=0.66(B=0.86)$ for the 10 -mm single-stage case and $\alpha=0.66(B=0.86)$ in the injector $/ \alpha \approx 0.78(B=0.49)$ in the accelerator for the two-stage case. From Equation (13), the dephasing length is $L_{\mathrm{dp}} \approx 23.1 \mathrm{~mm}$ for the 4-mm single-stage case, $L_{\mathrm{dp}} \approx 47.4 \mathrm{~mm}$ for the 10 -mm single-stage case and $L_{\mathrm{dp}} \approx$ $24.9 \mathrm{~mm}$ in the injector $/ L_{\mathrm{dp}} \approx 98.3 \mathrm{~mm}$ in the accelerator for the two-stage case. The pump depletion length due to pulse-front erosion is $L_{\mathrm{pd}} \approx 14.9 \mathrm{~mm}$ for the 4-mm singlestage case, $L_{\mathrm{pd}} \approx 24.1 \mathrm{~mm}$ for the 10 -mm single-stage case and $L_{\mathrm{pd}} \approx 15.7 \mathrm{~mm}$ in the injector/ $L_{\mathrm{pd}} \approx 39.2 \mathrm{~mm}$ in the accelerator for the two-stage case. From Equation (23), the beam energy is estimated to be $E_{b} \approx 353 \mathrm{MeV}$ with an effective acceleration length of $L_{\mathrm{acc}}=2 \mathrm{~mm}$ for the 4-mm single-stage case and $E_{b} \approx 1.02 \mathrm{GeV}$ with $L_{\mathrm{acc}}=8 \mathrm{~mm}$ for the 10-mm single-stage case. For the two-stage case, the output energy of the injector is $E_{b} \approx 409 \mathrm{MeV}$ with $L_{\mathrm{acc}} \approx 2.5 \mathrm{~mm}$ and the output energy of the accelerator stage reaches $E_{b} \approx 2.46 \mathrm{GeV}$ with $L_{\mathrm{acc}} \approx 10 \mathrm{~mm}$, assuming that the injection energy is $E_{b 0} \approx 409 \mathrm{MeV}$ and the focused spot size at the entrance of the accelerator stage is decreased to $r_{L}=7.5 \mu \mathrm{m}$ due to self-focusing in the injector stage, increasing the normalized vector potential up to $a_{0} \approx 10.4$. In this experiment, most of the charge produced in the injector is injected into the accelerator stage, while the large energy spread is attributed to the fact that the accelerator length is shorter than the dephasing length at which the energy compression takes place in the phase space as well as the maximum energy.

\section{Design of 10-GeV-level laser plasma accelerators}

At present, the most near-term prospects for $10-\mathrm{GeV}$-level laser plasma acceleration are confidently given by the scaling and methods described in the previous section. Here, we consider design examples of laser plasma accelerators capable of delivering $10-\mathrm{GeV}$ electron beams with bunch charges of $160 \mathrm{pC}\left(10^{9}\right.$ electrons per bunch) for three cases: a self-guided laser plasma accelerator in the bubble regime with $a_{0}=3$, a channel-guided laser plasma accelerator in the bubble regime with $a_{0}=2$ and a channel-guided laser plasma accelerator in the quasi-linear regime with $a_{0}=1.5$. For all three cases, we present design parameters for the laser and plasma for a driving laser wavelength of 800 $\mathrm{nm}$. Table 2 shows the design parameters of the $10-\mathrm{GeV}$ laser plasma accelerators for the abovementioned three cases and a $40-\mathrm{GeV}$ laser plasma accelerator with $a_{0}=2$ and a beam loading charge of $300 \mathrm{pC}$ by comparison with the results of 3D PIC simulation from the Lorentz-boosted frame OSIRIS code ${ }^{[16]}$. The design parameters for the $40-\mathrm{GeV}$ laser plasma accelerator are in good agreement with the PIC simulation results in terms of the operating plasma density, the accelerator length, i.e., the dephasing length, the matched spot radius and the matched power. The design formulas we used to evaluate these parameters are described in the following.

\subsection{Self-guided laser plasma accelerator in the bubble regime}

For a given energy gain $W$, the operating plasma density is determined from Equation (11) as

$$
\begin{aligned}
n_{e} & =\frac{2}{3} \alpha \kappa_{\mathrm{self}} a_{0} \frac{n_{c}}{\Delta \gamma_{\max }} \\
& \approx 5.94 \times 10^{16}\left[\mathrm{~cm}^{-3}\right] \kappa_{\mathrm{self}} a_{0}\left(\frac{0.8 \mu \mathrm{m}}{\lambda_{L}}\right)^{2}\left(\frac{10 \mathrm{GeV}}{W / \alpha}\right) .
\end{aligned}
$$

The accelerator length, equal to the dephasing length, becomes

$$
\begin{aligned}
L_{\mathrm{acc}} & =L_{\mathrm{dp}} \approx \sqrt{\frac{3}{2}} \frac{\left(\Delta \gamma_{\max } / \alpha\right)^{3 / 2}}{\pi a_{0} \kappa_{\mathrm{self}}^{1 / 2}} \lambda_{L} \\
& \approx \frac{0.9[\mathrm{~m}]}{a_{0} \kappa_{\mathrm{self}}^{1 / 2}}\left(\frac{\lambda_{L}}{0.8 \mu \mathrm{m}}\right)\left(\frac{W / \alpha}{10 \mathrm{GeV}}\right)^{3 / 2},
\end{aligned}
$$


Table 2. Design parameters for $10-\mathrm{GeV}$-level laser plasma accelerators in comparison with the results of the 3D PIC simulation ${ }^{[16]}$. Case A stands for the self-guided case in the bubble regime, designed by the formulas given in Section 4.1, case B for the self-guided case in the bubble regime, designed by the formulas given in Section 4.2, case $\mathrm{C}$ for the channel-guided case in the quasi-linear regime, designed by the formulas given in Section 4.3, and case D for the self-guided case in the bubble regime at $40 \mathrm{GeV}$, designed by the formulas given in Section 4.1.

\begin{tabular}{|c|c|c|c|c|c|}
\hline$\overline{\text { Case }}$ & $\overline{\mathrm{A}}$ & $\bar{B}$ & $\mathrm{C}$ & $\mathrm{D}$ & Ref. [16] \\
\hline$\overline{W(\mathrm{GeV})}$ & 10 & 10 & 10 & 40 & 38 \\
\hline$n_{e}\left(10^{17} \mathrm{~cm}^{-3}\right)$ & 1.9 & 2.7 & 1.1 & 0.28 & 0.22 \\
\hline$\Delta n / n_{0}(\%)$ & 0 & 5 & 5 & 0 & \\
\hline$L_{\mathrm{dp}}(\mathrm{m})$ & 0.38 & 0.42 & 0.87 & 4.7 & 5 \\
\hline$\lambda_{L}(\mathrm{~nm})$ & 800 & 800 & 800 & 800 & 800 \\
\hline$a_{0}$ & 3 & 2 & 1.5 & 2 & 2 \\
\hline$\kappa_{\mathrm{ch}}$ & 1.35 & 1.13 & 1.06 & 1.19 & \\
\hline$R_{m}$ & 2.3 & 2.9 & 3.6 & 3.2 & \\
\hline$r_{L}(\mu \mathrm{m})$ & 29 & 29 & 57 & 103 & 100 \\
\hline$\tau_{L}(\mathrm{fs})$ & 128 & 95 & 127 & 238 & 160 \\
\hline$P_{L}(\mathrm{TW})$ & 238 & 114 & 250 & 1483 & 1400 \\
\hline$P_{L} / P_{c}$ & 1.5 & 1.1 & 0.91 & 1.3 & 1.04 \\
\hline$U_{L}(\mathrm{~J})$ & 30 & 11 & 32 & 353 & 220 \\
\hline$Q_{b}(\mathrm{pC})$ & 160 & 160 & 160 & 300 & 300 \\
\hline $\mathrm{C}$ & 0.596 & 0.945 & 0.989 & 1.05 & \\
\hline$\alpha$ & 0.77 & 0.68 & 0.67 & 0.79 & \\
\hline
\end{tabular}

while the pump depletion length due to pulse-front erosion is given by $L_{\mathrm{pd}} \approx c \tau_{L} n_{c} / n_{e}$. The dephasing length should be less than the pump depletion length, i.e., $L_{\mathrm{pd}} \geqslant L_{\mathrm{dp}}$. Therefore, the pulse length is set to be $c \tau_{L} \geqslant(2 / 3 \pi) \sqrt{a_{0}} \kappa_{\text {self }} \lambda_{p}$.

$$
\begin{aligned}
\tau_{L} & \geqslant \sqrt{\frac{2}{3}} \frac{\lambda_{L}}{\pi c} \kappa_{\text {self }}^{1 / 2}\left(\frac{\Delta \gamma_{\max }}{\alpha}\right)^{1 / 2} \\
& \geqslant 97[\mathrm{fs}] \kappa_{\text {self }}^{1 / 2}\left(\frac{\lambda_{L}}{0.8 \mu \mathrm{m}}\right)\left(\frac{W / \alpha}{10 \mathrm{GeV}}\right)^{1 / 2} .
\end{aligned}
$$

The matched spot radius becomes

$$
\begin{aligned}
r_{L} & =\frac{1}{2 \pi} \sqrt{\frac{3}{2}} \lambda_{L} \frac{R_{m}}{\sqrt{a_{0} \kappa_{\mathrm{self}}}}\left(\frac{\Delta \gamma_{\mathrm{max}}}{\alpha}\right)^{1 / 2} \\
& \approx 22[\mu \mathrm{m}] \frac{R_{m}}{\sqrt{a_{0} \kappa_{\mathrm{self}}}}\left(\frac{\lambda_{L}}{0.8 \mu \mathrm{m}}\right)\left(\frac{W / \alpha}{10 \mathrm{GeV}}\right)^{1 / 2}
\end{aligned}
$$

where $R_{m}\left(a_{0}^{2}, 0\right)=k_{p} r_{L}$ is the dimensionless matched spot radius given by Equation (3) for self-guiding, $\Delta n / n_{0}=0$. The matched power is calculated as

$$
\begin{aligned}
P_{L} & =\frac{k_{p}^{2} r_{L}^{2} a_{0}^{2}}{32} P_{c}=\frac{51}{64}[\mathrm{GW}] \frac{a_{0} R_{m}^{2}}{\kappa_{\text {self }}} \frac{\Delta \gamma_{\text {max }}}{\alpha} \\
& \approx 15.6[\mathrm{TW}] \frac{a_{0} R_{m}^{2}}{\kappa_{\text {self }}}\left(\frac{W / \alpha}{10 \mathrm{GeV}}\right) .
\end{aligned}
$$

The required pulse energy is

$$
U_{L}=P_{L} \tau_{L} \geqslant 1.51[\mathrm{~J}] \frac{a_{0} R_{m}^{2}}{\kappa_{\text {self }}^{1 / 2}}\left(\frac{\lambda_{L}}{0.8 \mu \mathrm{m}}\right)\left(\frac{W / \alpha}{10 \mathrm{GeV}}\right)^{3 / 2}
$$

The field reduction factor $\alpha$ for loading charge $Q_{b}$ up to a given energy $W$ is obtained by solving the equation

$$
\alpha^{2}+C \alpha^{3 / 2}-1=0
$$

where the coefficient $C$ is given as

$$
C=\frac{Q_{b}}{312[\mathrm{pC}]} \frac{\kappa_{\mathrm{self}}^{1 / 2}}{k_{p}^{2} \sigma_{b}^{2}}\left(\frac{0.8 \mu \mathrm{m}}{\lambda_{L}}\right)\left(\frac{10 \mathrm{GeV}}{W}\right)^{1 / 2}
$$

\subsection{Channel-guided laser plasma accelerator in the bubble regime}

The operating plasma density is determined by

$$
\begin{aligned}
n_{e} & =2 \alpha \kappa_{\mathrm{ch}} a_{0} \frac{n_{c}}{\Delta \gamma_{\max }} \\
& \approx 1.78 \times 10^{17}\left[\mathrm{~cm}^{-3}\right] \kappa_{\mathrm{ch}} a_{0}\left(\frac{0.8 \mu \mathrm{m}}{\lambda_{L}}\right)^{2}\left(\frac{10 \mathrm{GeV}}{W / \alpha}\right),
\end{aligned}
$$

and the accelerator length becomes

$$
\begin{aligned}
L_{\text {stage }} & =L_{\mathrm{dp}} \approx \frac{\lambda_{L}}{\sqrt{2} \pi \kappa_{\mathrm{ch}}^{1 / 2} a_{0}}\left(\frac{\Delta \gamma_{\mathrm{max}}}{\alpha}\right)^{3 / 2} \\
& \approx \frac{0.5[\mathrm{~m}]}{\kappa_{\mathrm{ch}}^{1 / 2} a_{0}}\left(\frac{\lambda_{L}}{0.8 \mu \mathrm{m}}\right)\left(\frac{W / \alpha}{10 \mathrm{GeV}}\right)^{3 / 2} .
\end{aligned}
$$

The pump depletion length, at which the total field energy becomes half of the initial laser energy, is given by 


$$
\begin{aligned}
L_{\mathrm{pd}} & \approx \frac{\sqrt{\pi}}{2 \alpha^{2}} a_{0} \sigma_{L} \frac{n_{c}}{n_{e}}=\frac{1}{4} \sqrt{\frac{\pi}{\ln 2}} \frac{a_{0} c \tau_{L}}{\alpha^{2}} \frac{n_{c}}{n_{e}} \\
& \approx \frac{0.156[\mathrm{~m}]}{\alpha^{2} \kappa_{\mathrm{ch}}}\left(\frac{W / \alpha}{10 \mathrm{GeV}}\right)\left(\frac{\tau_{L}}{100 \mathrm{fs}}\right)
\end{aligned}
$$

where $\sigma_{L}=c \tau_{L} /(2 \sqrt{\ln 2}) \approx 0.6 c \tau_{L}$ is the rms pulse length. The requirement for the accelerator length $L_{\mathrm{acc}}=L_{\mathrm{dp}} \leqslant L_{\mathrm{pd}}$ bounds the minimum pulse duration as

$$
\begin{aligned}
& c \tau_{L} \geqslant \frac{8}{\pi}\left(\frac{\ln 2}{\pi}\right)^{1 / 2} \frac{\alpha^{2} \kappa_{\mathrm{ch}}}{\sqrt{a_{0}}} \lambda_{p} \quad \text { or } \\
& \tau_{L} \geqslant 320[\mathrm{fs}] \frac{\alpha^{2} \kappa_{\mathrm{ch}}^{1 / 2}}{a_{0}}\left(\frac{\lambda_{L}}{0.8 \mu \mathrm{m}}\right)\left(\frac{W / \alpha}{10 \mathrm{GeV}}\right)^{1 / 2} .
\end{aligned}
$$

The matched spot radius becomes

$$
\begin{aligned}
r_{L} & =\frac{\lambda_{L}}{2 \pi}\left(\frac{n_{c}}{n_{e}}\right)^{1 / 2} R_{m} \\
& \approx 12.6[\mu \mathrm{m}] \frac{R_{m}}{\sqrt{\kappa_{\mathrm{ch}} a_{0}}}\left(\frac{\lambda_{L}}{0.8 \mu \mathrm{m}}\right)\left(\frac{W / \alpha}{10 \mathrm{GeV}}\right)^{1 / 2},
\end{aligned}
$$

where $R_{m}\left(a_{0}^{2}, \Delta n / n_{0}\right)=k_{p} r_{L}$ is the dimensionless matched spot radius given by Equation (3). The matched power is calculated as

$$
P_{L}=\frac{k_{p}^{2} r_{L}^{2} a_{0}^{2}}{32} P_{c} \approx 5.2[\mathrm{TW}] \frac{a_{0} R_{m}^{2}}{\kappa_{\mathrm{ch}}}\left(\frac{W / \alpha}{10 \mathrm{GeV}}\right)
$$

and the required pulse energy is

$$
U_{L}=P_{L} \tau_{L} \geqslant 1.66[\mathrm{~J}] \frac{\alpha^{2} R_{m}^{2}}{\kappa_{\mathrm{ch}}^{1 / 2}}\left(\frac{\lambda_{L}}{0.8 \mu \mathrm{m}}\right)\left(\frac{W / \alpha}{10 \mathrm{GeV}}\right)^{3 / 2}
$$

The field reduction factor $\alpha$ is determined from the coefficient $C$, given by

$$
C \equiv \frac{Q_{b}}{180[\mathrm{pC}]} \frac{\kappa_{\mathrm{self}}^{1 / 2}}{k_{p}^{2} \sigma_{b}^{2}}\left(\frac{0.8 \mu \mathrm{m}}{\lambda_{L}}\right)\left(\frac{10 \mathrm{GeV}}{W}\right)^{1 / 2} .
$$

\subsection{Channel-guided laser plasma accelerator in the quasi-linear regime}

For a given $a_{0} \leqslant 1.7$, the pulse duration is given by

$$
\tau_{L} \approx \frac{160[\mathrm{fs}]}{a_{0} \sqrt{\kappa_{\mathrm{ch}}}}\left(\frac{\lambda_{L}}{0.8 \mu \mathrm{m}}\right)\left(\frac{W / \alpha}{10 \mathrm{GeV}}\right)^{1 / 2}
$$

For this pulse duration, the operating plasma density becomes

$$
n_{e} \approx 6.8 \times 10^{16}\left[\mathrm{~cm}^{-3}\right] \kappa_{\mathrm{ch}} a_{0}^{2}\left(\frac{0.8 \mu \mathrm{m}}{\lambda_{L}}\right)^{2}\left(\frac{10 \mathrm{GeV}}{W / \alpha}\right)
$$

Thus, the required accelerator length $L_{\mathrm{acc}}$ can be set to be

$$
L_{\mathrm{acc}}=L_{\mathrm{dp}} \approx \frac{1.65[\mathrm{~m}]}{a_{0}^{3} \kappa_{\mathrm{ch}}^{1 / 2}}\left(\frac{\lambda_{L}}{0.8 \mu \mathrm{m}}\right)\left(\frac{W / \alpha}{10 \mathrm{GeV}}\right)^{3 / 2},
$$

where $n_{c} / n_{e}$ is given by Equation (9). The matched spot radius is calculated from

$$
r_{L} \approx 20[\mu \mathrm{m}] \frac{R_{m}}{\kappa_{\mathrm{ch}}^{1 / 2} a_{0}}\left(\frac{\lambda_{L}}{0.8 \mu \mathrm{m}}\right)\left(\frac{W / \alpha}{10 \mathrm{GeV}}\right)^{1 / 2}
$$

where $R_{m}=k_{p} r_{L}$ is the dimensionless matched spot radius for a given $a_{0}$ and $\Delta n / n_{0}$, given by Equation (3). The required peak power of the laser pulse is given by

$$
P_{L}=\frac{k_{p}^{2} r_{L}^{2} a_{0}^{2}}{32} P_{c} \approx 13.7[\mathrm{TW}] \frac{R_{m}^{2}}{\kappa_{\mathrm{ch}}}\left(\frac{W / \alpha}{10 \mathrm{GeV}}\right)
$$

and the required pulse energy becomes

$$
U_{L}=P_{L} \tau_{L} \approx 2.2[\mathrm{~J}] \frac{R_{m}^{2}}{a_{0} \kappa_{\mathrm{ch}}^{3 / 2}}\left(\frac{\lambda_{L}}{0.8 \mu \mathrm{m}}\right)\left(\frac{W / \alpha}{10 \mathrm{GeV}}\right)^{3 / 2}
$$

The field reduction factor $\alpha$ is determined from the coefficient $C$, given by

$$
C \equiv \frac{Q_{b}}{111[\mathrm{pC}]} \frac{\kappa_{\mathrm{self}}^{1 / 2}}{a_{0} k_{p}^{2} \sigma_{b}^{2}}\left(\frac{0.8 \mu \mathrm{m}}{\lambda_{L}}\right)\left(\frac{10 \mathrm{GeV}}{W}\right)^{1 / 2} .
$$

\section{Conclusion}

We have provided an overview of recent progress in laser plasma accelerators from the perspective of experiments on the production of $\mathrm{GeV}$-level electron beams, and scaling formulas to describe energy gain for a self-guided laser plasma accelerator in the bubble regime $\left(a_{0} \geqslant 2\right)$, a channel-guided laser plasma accelerator in the bubble regime $\left(a_{0} \geqslant 2\right)$ and a channel-guided laser plasma accelerator in the quasi-linear regime $\left(a_{0}<2\right)$. Although most previous experiments have been focused on electron injection into the plasma bubble and the production of high-quality electron beams with small energy spread and emittance, employing a millimeter-scale gas jet, recent experimental results beyond $1-\mathrm{GeV}$ acceleration allow us to test the scaling formulas in depth, which are necessary for the design of the operating parameters of laser plasma accelerators to satisfy requirements such as energy gain and beam charge. Taking account of the group velocity correction factor $\kappa_{\mathrm{ch}}$ in the propagation of laser pulses with relativistic intensity, 
characterized by $a_{0}$, through plasma channels, including initially uniform plasma with $\Delta n / n_{0}=0$ and a preformed plasma channel with $\Delta n / n_{0} \neq 0$, provides the correct accelerator length equal to the dephasing length as well as the proper operating plasma density. Meanwhile, we found that the accelerating field reduction factor $\alpha$ due to beam loading can be properly evaluated by applying the resultant scaling formulas to recent experimental results for multi-GeV laser plasma accelerators ${ }^{[5,6]}$, leading to selfconsistent design parameters for the driving laser pulse achieving the requirement of beam energy and charge. Independently, we confirmed that the accelerating field reduction factor $\alpha$ can be obtained from the simple analytic formulas Equations (20)-(22), indicating good agreement with that obtained from 3D PIC simulation results ${ }^{[16,38]}$. A further detailed analysis on the beam loading effects such as bunch length and shape that affect the accelerating fields, energy gain and energy spread will be pursued in future work.

\section{Acknowledgements}

This work was supported by Project Code IBS-R012-D1. K. Nakajima was also supported by the National Natural Science Foundation of China (Project No. 51175324).

\section{References}

1. T. Tajima and J. M. Dawson, Phys. Rev. Lett. 43, 267 (1979).

2. W. P. Leemans, B. Nagler, A. J. Gonsalves, C. Toth, K Nakamura, C. G. R. Geddes, E. Esarey, C. B. Schroeder, and S. M. Hooker, Nat. Phys. 2, 696 (2006).

3. C. E. Clayton, J. E. Ralph, F. Albert, R. A. Fonseca, S. H. Glenzer, C. Joshi, W. Lu, K. A. Marsh, S. F. Martins, W. B. Mori, A. Pak, F. S. Tsung, B. B. Pollock, J. S. Ross, L. O. Silva, and D. H. Froula, Phys. Rev. Lett. 105, 105003 (2010).

4. H. Lu, M. Liu, W. Wang, C. Wang, J. Liu, A. Deng, J. Xu, C. Xia, W. Li, H. Zhang, X. Lu, C. Wang, J. Wang, X. Liang, Y. Leng, B. Shen, K. Nakajima, R. Li, and Z. Xu, Appl. Phys. Lett. 99, 091502 (2011).

5. X. Wang, R. Zgadzaj, N. Fazel, Z. Li, S. A. Yi, X. Zhang, W. Henderson, Y. Y. Chang, R. Korzekwa, H. E. Tsai, C. H. Pai, H. Quevedo, G. Dyer, E. Gaul, M. Martinez, A.C. Bernstein, T. Borger, M. Spinks, M. Donovan, V. Khudik, G. Shvets, T. Ditmire, and M. C. Downer, Nature Commun. 4, 1988 (2013).

6. H. T. Kim, K. H. Pae, H. J. Cha, I. J. Kim, T. J. Yu, J. H Sung, S. K. Lee, T. M. Jeong, and J. Lee, Phys. Rev. Lett. 111, 165002 (2013)

7. T. Kameshima, W. Hong, K. Sugiyama, X. Wen, Y. Wu, C. Tang, Q. Zhu, Y. Gu, B. Zhang, H. Peng, S.-i. Kurokawa, L. Chen, T. Tajima, T. Kumita, and K. Nakajima, Appl. Phys. Exp. 1, 066001 (2008).

8. S. Karsch, J. Osterhoff, A. Popp, T. P. Rowlands-Rees, Z. Major, M. Fuchs, B. Marx, R. Hörlein, K. Schmid, L. Veisz, S. Becker, U. Schramm, B. Hidding, G. Pretzler, D. Habs, F. Grüner, F. Krausz, and S. M. Hooker, New J. Phys. 9, 415 (2007).

9. O. Lundh, J. Lim, C. Rechatin, L. Ammoura, A. Ben-Ismail, X. Davoine, G. Gallot, J. P. Goddet, E. Lefebvre, V. Malka, and J. Faure, Nat. Phys. 7, 219 (2011).
10. N. A. M. Hafz, T. M. Jeong, I. W. Choi, S. K. Lee, K. H. Pae, V. V. Kulagin, J. H. Sung, T. J. Yu, K.-H. Hong, T. Hosokai, J. R. Cary, D.-K. Ko, and J. Lee, Nat. Photon. 2, 571 (2008).

11. J. S. Liu, C. Q. Xia, W. T. Wang, H. Y. Lu, C. Wang, A. H. Deng, W. T. Li, H. Zhang, X. Y. Liang, Y. X. Leng, X. M. Lu, C. Wang, J. Z. Wang, K. Nakajima, R. X. Li, and Z. Z. Xu, Phys. Rev. Lett. 107, 035001 (2011).

12. B. B. Pollock, C. E. Clayton, J. E. Ralph, F. Albert, A. Davidson, L. Divol, C. Filip, S. H. Glenzer, K. Herpoldt, W. Lu, K. A. Marsh, J. Meinecke, W. B. Mori, A. Pak, T. C. Rensink, J. S. Ross, J. Shaw, G. R. Tynan, C. Joshi, and D. H. Froula, Phys. Rev. Lett. 107, 045001 (2011).

13. A. Pak, K. A. Marsh, S. F. Martins, W. Lu, W. B. Mori, and C. Joshi, Phys. Rev. Lett. 104, 025003 (2010).

14. C. McGuffey, A. G. R. Thomas, W. Schumaker, T. Matsuoka, V. Chvykov, F. J. Dollar, G. Kalintchenko, V. Yanovsky, A. Maksimchuk, K. Krushelnick, V. Y. Bychenkov, I. V. Glazyrin, and A. V. Karpeev, Phys. Rev. Lett. 104, 025004 (2010).

15. C. Xia, J. Liu, W. Wang, H. Lu, W. Cheng, A. Deng, W. Li, H. Zhang, X. Liang, Y. Leng, X. Lu, C. Wang, J. Wang, K. Nakajima, R. Li, and Z. Xu, Phys. Plasmas 18, 113101 (2011).

16. S. F. Martins, R. A. Fonseca, W. Lu, W. B. Mori, and L. O. Silva, Nat. Phys. 6, 311 (2010)

17. J.-L. Vay, C. G. R. Geddes, E. Esarey, C. B. Schroeder, W. P. Leemans, E. Cormier-Miche, and D. P. Grote, Phys. Plasmas 18, 123103 (2011)

18. M. Tzoufras, C. Huang, J. H. Cooley, F. S. Tsung, J. Vieira, and W. B. Mori, J. Plasma Phys. 78, 401 (2012).

19. C. B. Schroeder, E. Esarey, C. G. R. Geddes, C. Benedetti, and W. P. Leemans, Phys. Rev. ST Accel. Beams 13, 101301 (2010)

20. K. Nakajima, A. Deng, X. Zhang, B. Shen, J. Liu, R. Li, Z. Xu, T. Ostermayr, S. Petrovics, C. Klier, K. Iqbal, H. Ruhl, and T. Tajima, Phys. Rev. ST Accel. Beams 14, 091301 (2011).

21. K. Nakajima, H. Lu, X. Zhao, B. Shen, R. Li, and Z. Xu, Chin. Opt. Lett. 11, 013501 (2013).

22. N. Blanchot, E. Bar, G. Behar, C. Bellet, D. Bigourd, F. Boubault, C. Chappuis, H. Coïc, C. Damiens-Dupont, O. Flour, O. Hartmann, L. Hilsz, E. Hugonnot, E. Lavastre, J. Luce, E. Mazataud, J. Neauport, S. Noailles, B. Remy, F. Sautarel, M. Sautet, and C. Rouyer, Opt. Express 18, 10088 (2010).

23. W. Leemans, C. G. R. Geddes, J. Faure, Cs. Tóth, J. van Tilborg, C. B. Schroeder, E. Esarey, and G. Fubiani, Phys. Rev. Lett. 91, 074802 (2003).

24. S. Kneip, C. McGuffey, J. L. Martins, S. F. Martins, C. Bellei, V. Chvykov, F. Dollar, R. Fonseca, C. Huntington, G. Kalintchenko, A. Maksimchuk, S. P. D. Mangles, T. Matsuoka, S. R. Nagel, C. A. J. Palmer, J. Schreiber, K. Ta Phuoc, A. G. R. Thomas, V. Yanovsky, L. O. Silva, K. Krushelnick, and Z. Najmudin, Nat. Phys. 6, 980 (2010).

25. K. Nakajima, M. Kando, T. Kawakubo, T. Nakanishi, and A. Ogata, Nucl. Instrum. Methods A 375, 593 (1996).

26. F. Grüner, S. Becker, U. Schramm, T. Eichner, M. Fuchs, R. Weingartner, D. Habs, J. Meyer-Ter-Vehn, M. Geissler, M. Ferrario, L. Serafini, B. Van der Geer, H. Backe, W. Lauth, and S. Reiche, Appl. Phys. B 86, 431 (2007).

27. K. Nakajima, Nat. Phys. 4, 92 (2008).

28. K. Nakajima, A. H. Deng, H. Yoshitama, Nasr A. M. Hafz, H. Y. Lu, B. F. Shen, J. S. Liu, R. X. Li, and Z. Z. Xu, Free Electron Laser S. Varró, ed. Vol. 119 (InTech, Rijeka, Croatia, 2012), Ch. 5. 
29. K. Nakajima, D. Fisher, D. T. Kawakubo, H. Nakanishi, A. Ogata, Y. Kato, Y. Kitagawa, R. Kodama, K. Mima, H. Shiraga, K. Suzuki, K. Yamakawa, T. Zhang, Y. Sakawa, T. Shoji, Y. Nishida, N. Yugami, M. Downer, and T. Tajima, Phys. Rev. Lett. 74, 4428 (1995).

30. A. Modena, Z. Najmudin, A. E. Dangor, C. E. Clayton, K. A. Marsh, C. Joshi, V. Malka, C. B. Darrow, C. Danson, D. Neely, and F. N. Walsh, Nature 377, 606 (1995).

31. N. E. Andreev, L. M. Gorbunov, V. I. Kirsanov, A. A. Pogosova, and R. R. Ramazashvili, Sov. Phys. JETP 55, 571 (1992).

32. Z. Najmudin, K. Krushelnick, E. L. Clark, S. P. D. Mangles, B. Walton, A. E. Dangor, S. Fritzler, V. Malka, E. Lefebvre, D. Gordon, F. S. Tsung, and C. Joshi, Phys. Plasmas 10, 2071 (2003).

33. V. Malka, S. Fritzler, E. Lefebvre, M.-M. Aleonard, F. Burgy, J.-P. Chambaret, J.-F. Chemin, K. Krushelnick, G. Malka, S. P. D. Mangles, Z. Najmudin, M. Pittman, J.-P. Rousseau, J.-N. Scheurer, B. Walton, and A. E. Dangor, Science 298, 1596 (2002).

34. S. P. D. Mangles, C. D. Murphy, Z. Najmudin, A. G. R. Thomas, J. L. Collier, A. E. Dangor, E. J. Divall, P. S. Foster, J. G. Gallacher, C. J. Hooker, D. A. Jaroszynski, A. J. Langley, W. B. Mori, P. A. Norreys, F. S. Tsung, R. Viskup, B. R. Walton, and K. Krushelnick, Nature 431, 535 (2004).

35. C. G. R. Geddes, C. Toth, J. van Tilborg, E. Esarey, C. B. Schroeder, D. Bruhwiler, C. Nieter, J. Cary, and W. P. Leemans, Nature 431, 538 (2004).

36. J. Faure, Y. Glinec, A. Pukhov, S. Kiselev, S. Gordienko, E. Lefebvre, J. P. Rousseau, F. Burgy, and V. Malka, Nature 431, 541 (2004).

37. I. Kostyukov, A. Pukhov, and S. Kiselev, Phys. Plasmas 11, $5256(2004)$

38. W. Lu, M. Tzoufras, C. Joshi, F. S. Tsung, W. B. Mori, J. Vieira, R. A. Fonseca, and L. O. Silva, Phys. Rev. ST Accel. Beams 10, 061301 (2007)

39. S. Kalmykov, A. Yi, V. Khudik, and G. Shvets, Phys. Rev. Lett. 103, 135004 (2009).

40. J. Faure, C. Rechatin, A. Norlin, A. Lifschitz, Y. Glinec, and V. Malka, Nature 444, 737 (2006)

41. H. Kotaki, I. Daito, M. Kando, Y. Hayashi, K. Kawase, T. Kameshima, Y. Fukuda, T. Homma, J. Ma, L. M. Chen, T. Z. Esirkepov, A. S. Pirozhkov, J. K. Koga, A. Faenov, T. Pikuz, H. Kiriyama, H. Okada, T. Shimomura, Y. Nakai, M. Tanoue, H. Sasao, D. Wakai, H. Matsuura, S. Kondo, S. Kanazawa, A. Sugiyama, H. Daido, and S. V. Bulanov, Phys. Rev. Lett. 103, 194803 (2009).
42. K. Schmid, A. Buck, C. M. S. Sears, J. M. Mikhailova, R. Tautz, D. Herrmann, M. Geissler, F. Krausz, and L. Veisz, Phys. Rev. ST Accel. Beams 13, 091301 (2010).

43. A. J. Gonsalves, K. Nakamura, C. Lin, D. Panasenko, S. Shiraishi, T. Sokollik, C. Benedetti, C. B. Schroeder, C. G. R. Geddes, J. van Tilborg, J. Osterhoff, E. Esarey, C. Toth, and W. P. Leemans, Nat. Phys. 7, 862 (2011).

44. Y. Ehrlich, C. Cohen, A. Zigler, J. Krall, P. Sprangle, and E. Esarey, Phys. Rev. Lett. 77, 4186 (1996).

45. N. M. Naumova, J. Koga, K. Nakajima, T. Tajima, T. Zh. Esirkepov, S. V. Bulanov, and F. Pegoraro, Phys. Plasma 8, 4149 (2001).

46. L. M. Chen, H. Kotaki, K. Nakajima, J. Koga, S. V. Bulanov, T. Tajima, Y. Q. Gu, H. S. Peng, X. X. Wang, T. S. Wen, H. J. Liu, C. Y. Jiao, C. G. Zhang, X. J. Huang, Y. Guo, K. N. Zhou, J. F. Hua, W. M. An, C. X. Tang, and Y. Z. Lin, Phys. Plasmas 14, 040703 (2007).

47. R. F. Hubbard, D. Kaganovich, B. Hafizi, C. I. Moore, P. Sprangle, A. Ting, and A. Zigler, Phys. Rev. E 63, 036502 (2001).

48. B. Hafizi, A. Ting, P. Sprangle, and R. F. Hubbard, Phys. Rev. E 62, 4120 (2000).

49. B. Hafizi, A. Ting, R. F. Hubbard, P. Sprangle, and J. R. Penano, Phys. Plasmas 10, 1483 (2003).

50. C. Chiu, S. Cheshkov, and T. Tajima, Phys. Rev. ST Accel. Beams 3, 101301 (2000).

51. S. Kneip, S. R. Nagel, S. F. Martins, S. P. D. Mangles, C. Bellei, O. Chekhlov, R. J. Clarke, N. Delerue, E. J. Divall, G. Doucas, K. Ertel, F. Fiuza, R. Fonseca, P. Foster, S. J. Hawkes, C. J. Hooker, K. Krushelnick, W. B. Mori, C. A. J. Palmer, K. Ta Phuoc, P. P. Rajeev, J. Schreiber, M. J. V. Streeter, D. Urner, J. Vieira, L. O. Silva, and Z. Najmudin, Phys. Rev. Lett. 103, 035002 (2009).

52. D. H. Froula, C. E. Clayton, T. Döppner, K. A. Marsh, C. P. J. Barty, L. Divol, R. A. Fonseca, S. H. Glenzer, C. Joshi, W. Lu, S. F. Martins, P. Michel, W. B. Mori, J. P. Palastro, B. B. Pollock, A. Pak, J. E. Ralph, J. S. Ross, C.W. Siders, L. O. Silva, and T. Wang, Phys. Rev. Lett. 103, 215006 (2009).

53. T. P. A. Ibbotson, N. Bourgeois, T. P. Rowlands-Rees, L. S. Caballero, S. I. Bajlekov, P. A. Walker, S. Kneip, S. P. D. Mangles, S. R. Nagel, C. A. J. Palmer, N. Delerue, G. Doucas, D. Urner, O. Chekhlov, R. J. Clarke, E. Divall, K. Ertel, P. S. Foster, S. J. Hawkes, C. J. Hooker, B. Parry, P. P. Rajeev, M. J. V. Streeter, and S. M. Hooker, Phys. Rev. ST Accel. Beams 13, 031301 (2010). 